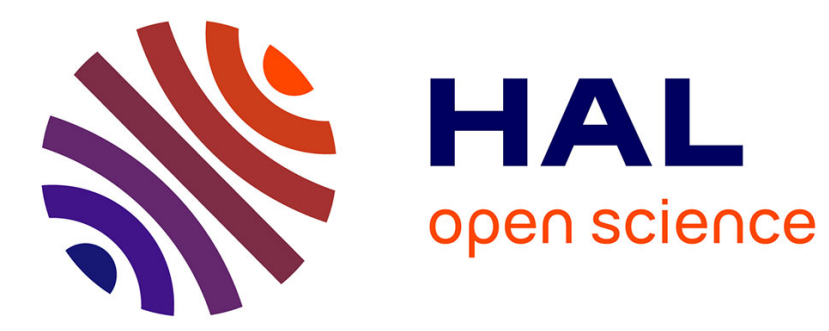

\title{
JPEG based Compression of Digital Holograms
}

Nada Chamakhi, Ines Bouzidi, Azza Ouled Zaid, Frédéric Dufaux

\section{To cite this version:}

Nada Chamakhi, Ines Bouzidi, Azza Ouled Zaid, Frédéric Dufaux. JPEG based Compression of Digital Holograms. 7th European Workshop on Visual Information Processing (EUVIP 2018), Nov 2018, Tampere, Finland. pp.1-6, 10.1109/EUVIP.2018.8611713 . hal-01879327

\section{HAL Id: hal-01879327 https://hal.science/hal-01879327}

Submitted on 10 Jan 2020

HAL is a multi-disciplinary open access archive for the deposit and dissemination of scientific research documents, whether they are published or not. The documents may come from teaching and research institutions in France or abroad, or from public or private research centers.
L'archive ouverte pluridisciplinaire HAL, est destinée au dépôt et à la diffusion de documents scientifiques de niveau recherche, publiés ou non, émanant des établissements d'enseignement et de recherche français ou étrangers, des laboratoires publics ou privés. 


\section{JPEG based Compression of Digital Holograms}

\author{
Nada Chamakhi \\ Institut Supérieur d'Informatique \\ Université de Tunis El Manar \\ Ariana, Tunisia
}

\author{
Ines Bouzidi \\ and Azza Ouled Zaid \\ Lab. Systèmes de Communications \\ ENIT - Université de Tunis El Manar \\ Tunis, Tunisia \\ azza.ouledzaid@isi.rnu.tn
}

\author{
Frédéric Dufaux \\ Lab. des Signaux et Systèmes (L2S) \\ CNRS - CentraleSupelec - Univ. Paris-Sud \\ Gif-sur-Yvette, France \\ frederic.dufaux@12s.centralesupelec.fr
}

\begin{abstract}
Modern holography for 3D imaging allows to reconstruct all the parallaxes that are needed for a truly immersive visualisation. Nevertheless, it represents a huge amount of data which induces higher transmission and storage requirements. To gain more popularity and acceptance, digital holography demands development of efficient coding schemes that provide significant data compression at low computation cost. Another issue that needs to be tackled when designing holography coding algorithms is interoperability with commonly used formats. The upcoming JPEG Pleno standard aims to develop a standard framework for the representation and exchange of new imaging modalities, such as holographic imaging, while maintaining backward compatibility with legacy JPEG decoders. This paper presents a lossy compression method for holographic images that exhibits good coding performance while considering the computation cost and backward compatibility with legacy JPEG standard. To validate our findings, the results of our tests are shown and interpreted.
\end{abstract}

Index Terms-Holography, lossy compression, JPEG, angular spectrum reconstruction

\section{INTRODUCTION}

Nowadays, static planar-like images are giving place to more informative volume-like images, thereby providing viewers an immersive experience. Among these volume-like images, the concept of stereoscopic representation is widely used in movie theatres and high end televisions. But this $3 \mathrm{D}$ visualization technology only exploits limited depth cues and causes visual fatigue due to the inherent accommodationvergence conflict. These limitations are surpassed when using holographic systems. Indeed, holographic visualisation guarantees that the convergence and focus points are the same as in real life, which makes it possible to have the most realistic depth perception. Thus, holography can be considered as a unique imaging technique which promises to change the way $3 \mathrm{D}$ visual information will be generated and consumed in the future, defeating all limitations and constraints of current 3D technologies.

The optical holography has been initiated in 1947 by Denis Gabor to eliminate aberrations in electron microscopy by recording and reconstructing amplitude and phase of a wave field [1]. Since then, many techniques have been developed to reconstruct and record holograms with the same aim of providing simpler solutions and higher quality [2]. The physical concept of light propagation can be modelled and simulated on a computer, enabling the production of computer generated holograms (CGHs) [3], [4] which have the advantage to avoid the need for a physical holographic recording set-up.

The visual richness of digital holograms is naturally associated to large amounts of data, which means huge storage and transmission bandwidth requirements. Consequently, the issue of efficiently compressing this type of data is a major concern. Hologram compression differs to image compression principally because holograms encode $3 \mathrm{D}$ information in complex-valued pixels and secondly because of their speckle nature. Due to the fact that holographic data represents the diffraction patterns that correspond to $3 \mathrm{D}$ scene information as intensity and/or phase over a bidirectional plane, the majority of research work on holographic data compression have focused on the extension of conventional image/video coding standards and algorithms. Fundamentally there are two modes of image compression: lossless and lossy compression. Lossless compression techniques have been already performed on digital holographic data [5]. Even if perfect reconstruction is to be attained, reversible compression methods are usually inefficient for holographic data [7]. This is principally due to the low spatial redundancies of hologram's speckle nature. Therefore, lossy compression of holographic data still play a critical role in reducing the storage and transmission costs [8].

To date, there is no coding standard appropriate for holographic images. Various reviews, summarizing the pioneering works on holographic data compression were published [9], [10], [12], [11], [13]. On the other hand, the upcoming JPEG Pleno standard is actually working on the issue of high efficiency coding of plenoptic images and digital holographic data while maintaining backward compatibility with legacy JPEG decoders [14]. It is evident that a common representation framework, which is backward compatible with JPEG would provide interoperability that might enable a wider use of this new type of image modalities without breaking the current JPEG ecosystem.

The aim of this paper is to study the effectiveness of JPEG based solution for DH compression. The main idea consists in using a rate-control compression algorithm, which uses an optimized bit allocation mechanism while guaranteeing a full compliance with the JPEG bitstream. The performance evaluation study includes the reconstruction quality and the computational complexity.

The paper is structured as follows. Section II focuses on the 


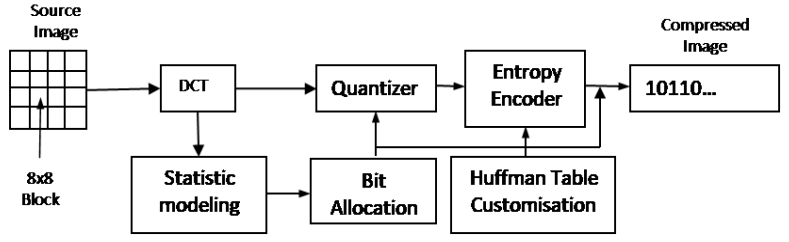

Fig. 1. Block diagram for the JPEG-OPT coding chain.

description of the proposed compression algorithm, namely JPEG-OPT [15]. Section III assesses the performance of the aforementioned coding algorithm when using holographic data. Finally, Section IV presents the conclusions.

\section{DESCRIPTION OF JPEG-OPT ALGORITHM}

As cited earlier, the objective of this work is to study the effectiveness of JPEG-based coding algorithm, named JPEGOPT, for holographic data while considering the compression performance and computation cost. JPEG-OPT has the advantage of ensuring near-optimal rate control in DCT-based compression while guaranteeing backward compatibility with JPEG legacy format. The subsequent algorithmic parts of the JPEG-OPT compression system are illustrated in a block diagram form as shown in Fig. 1.

Let us designate an $H \times W$ scaled holographic data by a set $I$ of $\frac{H \times W}{64}$ blocks. For simplicity purpose, the $8 \times 8$ set of spatial frequencies, in the DCT-domain is ordered into a 1-D array of 64 coefficients using the zig-zag scan. A DCT coefficient with coordinates $(i, j),(i, j=1, \ldots, 8)$, is then indexed by $n=8 \times i+j$. The $n^{\text {th }}$ component belonging to the DCT block $C^{b}$ is then denoted by $C[n]^{b}$, where $n$ is the coefficient position and $b$ is the block index.

Before passing through the Huffman entropy coder, the DCT indices undergo run-length coding, which combines zero DCT indices from different frequency bins into one symbol.

The JPEG syntax leaves the selection of the quantization step sizes to the encoder. Unlike baseline JPEG encoder, JPEG-OPT uses a rate-distortion optimization scheme to obtain an efficient image-adaptive quantization table. The ratedistortion optimization problem is to find a quantization table $Q$, such that the rate $R(Q) \leq R^{*}$, and $D(Q)$ is minimized, for a given rate budget $R^{*}$.

Because of the zero run-length coding, it is difficult to obtain a solution to the rate-distortion optimisation problem with classical bit allocation methods. Ratnakar and Miron Livny [15] proposed a sub-optimal solution to solve the quantization table design problem using histograms of the probability distribution of the DCT coefficients over all the blocks.

Based on Parseval theorem, the total distortion that affects an input block $I^{b}$ can be replaced by the sum of the coefficient distortions in its corresponding DCT coefficient block $C^{b}$. On the other hand, the bit rate can be estimated as the sum (over the 64 frequency localizations) of the entropies of the quantized DCT coefficients over all the blocks. Given a quantization table $Q$, let $D(Q)$ and $R(Q)$ represent the
Mean square error (MSE) and estimated bit rate (in bits per sample), respectively, resulting from compression of a scaled holographic data using the quantization table $Q$. For a given quantizer step size $q, D[n][q]$, the contribution to total distortion at the $n^{t h}$ frequency position is given by:

$$
D[n][q]=\frac{1}{64} \operatorname{Mean}\left(\left(C[n]-\operatorname{Round}\left(\frac{C[n]}{q}\right)\right)^{2}\right),
$$

where the "Mean" is taken over all the $8 \times 8$ blocks in the quantized and transformed domain. Similarly, the rate contribution, $R[n]\left[q_{n}\right]$, at the $n^{t h}$ frequency position is estimated by:

$$
R[n][q]=\operatorname{Entropy}\left(\operatorname{Round}\left(\frac{C[n]}{q}\right)\right),
$$

$R[n][q]$ is calculated using the histogram of DCT coefficient distribution in the $n^{t h}$ frequency position.

For a specific quantization table $Q$, the modeled bit-rate is given by:

$$
R(Q)=\sum_{n=0}^{63} R[n][Q[n]],
$$

whereas the distortion is estimated by:

$$
D(Q)=\sum_{n=0}^{63} D[n][Q[n]] .
$$

Giving $Q_{m}$ and $Q_{M}$ the quantization tables that, respectively, represent the minimum and maximum values possible for each quantization table entry, such that, $Q_{m}[n] \prec Q[n] \leq$ $Q_{M}[n]$. The rate-distortion optimisation scheme builds tables of $D[n][q]$ and $R[n][q]$, for each quantizer step size $q=Q[n]$ in the predefined operating range.

The rate-distortion optimisation problem is then formulated by Lagrangian minimization, $\min (D(Q)+\lambda R(Q))$, in such manner that solutions of the latter for any non-negative $\lambda$ are solutions to the former for a target bit rate $R^{*}$. Using both Eqs 3 and 4, the Lagrangian minimisation problem can be expressed by:

$$
\sum_{n=0}^{63} D[n][Q[n]]+\lambda R[n][Q[n]] .
$$

For each frequency localization $n$, a subset of the operating points $(R[n][],. D[n][]$.$) are pruned and sorted such that R[n][$. $]$ is strictly decreasing, whereas $D[n][$.$] is strictly increasing.$ The Graham scan algorithm [6] is then performed to get the (lower half of) convex hull of the $(R[n][],. D[n][]$.$) points.$ The Graham algorithm begins at the left-most point (which is certainly on the convex hull), and then retains points in increasing order of distortion. If the line segment from the previous point to the new point is a right turn with respect to the last such line segment, in that case the previous point is removed from the hull. The removals continue until a left turn is feasible. Let $h_{n}$ designate the remaining points on the hull and $\left\{q_{n}(1), \ldots, q_{n}\left(h_{n}\right)\right\}$ the corresponding quantizer values. 
TABLE I

PARAMETERS OF CGH GENERATION SETUPS.

\begin{tabular}{|c|c|c|c|c|c|}
\hline \multirow{2}{*}{ Database } & \multirow{2}{*}{ Hologram } & \multicolumn{4}{|c|}{$\begin{array}{l}\text { Specifications of the different } \\
\text { generation setups }\end{array}$} \\
\cline { 3 - 6 } & & $\begin{array}{l}\text { Resolution } \\
\text { (pixels) }\end{array}$ & $\begin{array}{l}\text { Pixel } \\
\text { pitch } \\
(\mu \mathrm{m})\end{array}$ & $\begin{array}{l}\text { Recons- } \\
\text { truction } \\
\text { distance } \\
(\mathrm{cm})\end{array}$ & $\begin{array}{l}\text { Wave } \\
\text { length } \\
(\mathrm{nm})\end{array}$ \\
\hline \hline $\begin{array}{c}\text { Télécom } \\
\text { ParisTech }\end{array}$ & Girl & $512 \times 512$ & 6.33 & 6.48 & 633 \\
\hline Interfere-I & 3D Venus & $1920 \times 1080$ & 8 & 0.51 & 632.8 \\
\hline $\begin{array}{l}\text { Emerglmg } \\
\text { HoloGrail }\end{array}$ & Horse & $968 \times 968$ & 4.4 & 14 & 632.8 \\
\hline
\end{tabular}

The slopes of the rate-distortion curve for the $n^{t h}$ coefficients at these $h_{n}$ points are given by:

$$
\lambda_{n}(k)=\frac{R_{n}\left(q_{n}(k)\right)-R_{n}\left(q_{n}(k+1)\right)}{D_{n}\left(q_{n}(k+1)\right)-D_{n}\left(q_{n}(k)\right)}
$$

for $k=1,2, \ldots, h_{n}-1$ and $\lambda_{n}\left(h_{n}\right)=0$. Hence, $\lambda_{n}(k)>$ $\lambda_{n}(k)$, for $k=1,2, \ldots, h_{n}-1$. Notably, for any given $\lambda>0$, the Lagrangian, $D[n][q]+\lambda R[n][q]$ is minimized for $q=q_{n}(k)$, with $k$ being the least index for which $\lambda>\lambda_{n}(k)$. More generally, for any given $\lambda>0$, the 64 binary searches can be used to find the quantizer values, belonging to the operating range, such that $R(Q)+\lambda D(Q)=$ $\sum_{n=0}^{63} D[n][Q[n]]+\lambda R[n][Q[n]]$ is minimized. If a target bit rate $R^{*}$ is considered, the bisection method is used to easily search for a $\lambda$ that would satisfy the target with respect to the fixed tolerance.

To have a better understanding of the effectiveness of JPEGOPT compression method in the context of digital holograms, experiments have been conducted to evaluate its efficiency.

\section{Performance evaluation}

Experiments have been conducted to evaluate JPEG-OPT coding scheme in the context of lossy compression of digital holograms. To complete our study, comparisons have been made with reference methods, on the basis of bit rate, reconstruction error and computational cost.

\section{A. Experimental setup}

Due to space limitation, our simulations have been carried out using three CGHs (Girl, 3D Venus and Horse) selected from three databases: Télécom ParisTech, InterfereI and EmergIMG. The first holographic content dataset was developed in the framework of the PhD research work of Y. Xing at Télécom ParisTech (see [9]). The Interfere-I dataset was made available by Prof. Peter Schelkens from ETRO-VUB and is available at http://www.erc-interfere.eu./index.html. The EmergIMG database was made available by Prof. Manuela Pereira from University of Beira Interior, Covilh and is available at http://emergimg.di.ubi.pt/downloads.html. The basic setups, for the considered holograms are summarized in Table I. As mentioned in [10], the interferograms can be represented using various formatting options. Among these we use shifted distance data (D1/D2) and Real/Imaginary (Rea/Ima) representations. Note that because of the chaotic nature of Amplitude/Phase format, the use of such data is unbeneficial when targeting compression. Also, the three interferograms intensities format requires more components to represent a hologram and thus it is evidently the least efficient format for the compression purpose.

For the assessment purpose, objective comparisons between JPEG-OPT, JPEG [16], QT-L [17], JPEG 2000 (using Kakadu implementation) [18], SPIHT [19] and HEVC [20] have been conducted to evaluate the quality of the decoded holograms in the reconstruction domain. JPEG 2000, QT-L and SPIHT are wavelet-based compression techniques. The first two codecs exploit the intra-band dependencies between wavelet coefficients, whereas the third exploits the inter-band dependencies between the wavelet sub-bands and employs a zero-tree structure to drop the insignificant coefficients. To offer a fair comparison, the tested wavelet-based coders used the following set of parameters: irreversible $(9,7)$ filter-bank; 3 levels of dyadic wavelet decomposition; and a single layer not considering the progressive decoding mode. It is noteworthy that YUV format is the main raw video format used in video coding softwares. Particularly, the Main Still Picture profile in HEVC software only accepts YUV 4:2:0 format as input. So, before HEVC encoding, the digital holograms were converted to YUV 4:2:0 format to satisfy the unique input format supported by the reference software.

The Angular Spectrum Method (ASM) has been used to perform the reconstruction process for Girl and 3D Venus, whereas Fresnel diffraction Method (FDM) has been used to perform the reconstruction for Horse. It is worth noting that the ASM did not work on Horse because the object is substantially bigger than the hologram. Since angular spectrum preserves pixel pitch, it will not be able to fully show the object. On the other hand, by using numerical Fresnel diffraction (the Fourier form), the pixel pitch will change depending on the propagation distance, and it will grow so we can fully view the entire hologram. The softwares of both ASM and FDM methods have been kindly provided by Prof. Manuela Pereira from University of Beira Interior, Covilha in Portugal. In the proposed assessment methodology, the reconstructions associated to the original and decoded holographic data are compared by means of BD-PSNR using BjØntegaard model [21]. In this case of study, the bit rate is the sum of the bit rates for the two compressed holographic data components. The BD-PSNR metric receives two sets of Rate-Distortion (RD) points defining two RD performance curves and measures the average difference in $\mathrm{dB}$ between the PSNR values for a same rate.

In order to evaluate the time complexity of the tested codecs, execution-time tests were conducted employing 32-bit $\mathrm{C}++$ executables generated by Visual Studio 10.0 operating in release mode. The time-complexity evaluation for JPEG 2000, QT-L and SPIHT was carried out using the executables that are made publicly available by the authors. 
TABLE II

COMPRESSION PERFORMANCE EVALUATION (IN BD-PSNR [DB] REGARDING HEVC INTRA) WHEN USING THE RECONSTRUCTED HOLOGRAMS.

\begin{tabular}{|c|c|c|c|c|c|c|}
\hline \multirow{2}{*}{ Model } & Format & $\begin{array}{c}\text { BD-PSNR } \\
\text { (HEVC-JPEG-OPT) }\end{array}$ & $\begin{array}{c}\text { BD-PSNR } \\
\text { (HEVC-JPEG2000) }\end{array}$ & $\begin{array}{c}\text { BD-PSNR } \\
\text { (HEVC-QT-L) }\end{array}$ & $\begin{array}{c}\text { BD-PSNR } \\
\text { (HEVC-SPIHT) }\end{array}$ & $\begin{array}{c}\text { BD-PSNR } \\
\text { (HEVC-JPEG) }\end{array}$ \\
\hline 3DVenus & D1/D2 & 2.102 & 2.265 & 2.074 & 11.976 & 3.493 \\
$1920 \times 1080$ & Rea/Ima & 2.298 & 2.555 & 1.493 & 12.412 & 3.883 \\
\hline Girl & D1/D2 & 5.346 & 2.871 & 5.540 & 3.106 & 8.131 \\
$512 \times 512$ & Rea/Ima & 5.962 & 3.844 & 4.967 & 4.655 & 7.410 \\
\hline Horse & D1/D2 & 0.658 & 1.338 & 1.092 & 1.107 & 1.576 \\
$968 \times 968$ & Rea/Ima & -3.325 & -2.518 & -2.784 & -2.735 & -2.634 \\
\hline
\end{tabular}

\section{B. Performance assessment in the reconstruction Domain}

In this section, we evaluate the compression performance of JPEG-OPT, JPEG 2000, QT-L, SPIHT and JPEG in the reconstruction domain. The results of our experiments in terms of BjØntegaard Delta-PSNR, when taking HEVC as a reference codec, are summarized in Table II.

Taking the Delta-PSNR to serve as a metric to compare the coding efficiency in the reconstruction domain, the gap between the average Delta-PSNR values using Real/Imaginary and shifted distance data representations for 3D Venus and Girl varies is $0.146 \mathrm{~dB}$ and $0.47 \mathrm{~dB}$, respectively, which is relatively low. One may conclude that the performances of the Real/Imaginary components have results very close to those obtained for the shifted distances components. The results also reveal that HEVC significantly outperforms all the tested codecs, except for Real/Imaginary representation of Horse object, where HEVC yields lower performance than the other tested codecs. One may thus conclude that the speckle noise in Horse object has a greater impact on HEVC efficiency.

Examining the efficiency of JPEG-OPT, JPEG 2000, QT-L, SPIHT and JPEG, we observe that, for Girl object, JPEG 2000 performs best, followed by SPIHT. On the other hand, QT-L yields superior performance for 3D Venus whereas JPEG-OPT systematically yields superior reconstruction quality for Horse object. One may also notice that for the same object, both QT-L and SPIHT offer roughly similar performance despite the fact that QT-L is an intra-band coding method whereas SPIHT is an inter-band coding method. More generally, the reported average Delta-PSNR results show that JPEG-OPT produces satisfactory results which are competitive with those provided by JPEG 2000.

In Fig. 2, visual comparisons between JPEG-OPT and HEVC are provided using the reconstructed versions of 3D Venus and Girl object. Examining these renderings, we can clearly observe that for Real/Imaginary representation, JPEGOPT architecture provides a visually appealing quality even for low bit rate. Besides, one may notice that both JPEG-OPT and HEVC methods are able to produce very similar results despite the difference in PSNR in favour of HEVC.

\section{Computation times}

Table III provides the coding and decoding execution time, in second (s), as a function of the tested representation formats, for 3D Venus, Girl and Horse holograms. Before discussing the

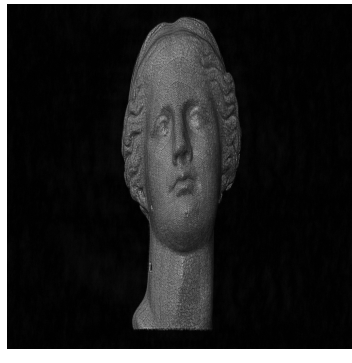

(a)

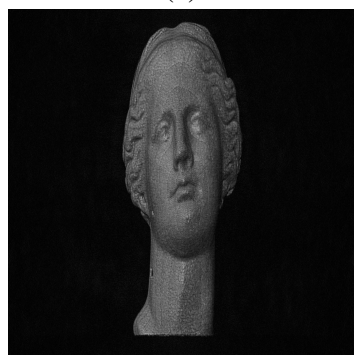

$\mathrm{PSNR}=27.37 \mathrm{~dB}$

(c)

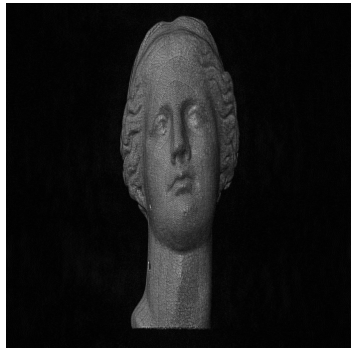

$\mathrm{PSNR}=28.59 \mathrm{~dB}$

(e)

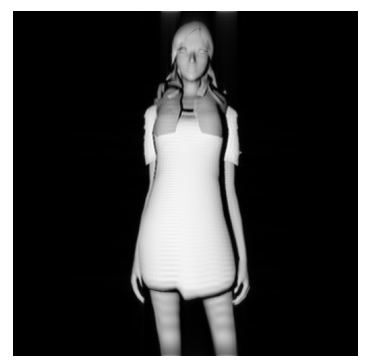

(b)

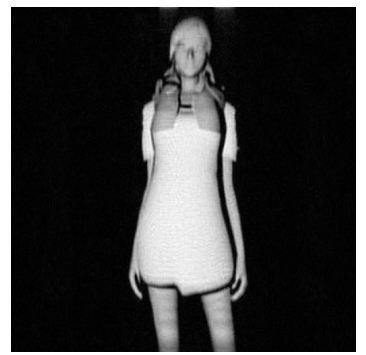

$\mathrm{PSNR}=33.63 \mathrm{~dB}$

(d)

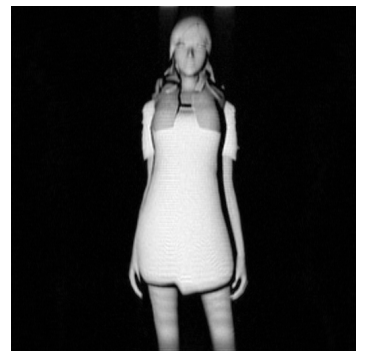

$\mathrm{PSNR}=39.24 \mathrm{~dB}$

(f)
Fig. 2. Visual comparison of the reconstructed 3D Venus and Girl objects (real/imaginary format) after being compressed at $0.4 \mathrm{bpp}$, using (c-d) JPEGOPT and (e-f) HEVC. Reconstructions of the original objects are shown in (a-b)

results of the complexity assessments, we would like to emphasize the fact that the timings reported in Table III are very implementation dependant. But, they allow to know whether the order of complexity of the tested technique is acceptable or not. To prevent possible external processes from affecting the result, For each test condition, the runs were repeated 10 times and the average running time of repeated execution is retained. From the reported results, one may notice that, for 
TABLE III

EXECUTION TIMES (IN SECONDS) FOR CODING THE HOLOGRAMS IN THE TEST SET.

\begin{tabular}{|c|c|c|c|c|c|c|c|c|c|c|}
\hline \multirow{2}{*}{ Model } & \multicolumn{2}{|c|}{ JPEG-OPT } & \multicolumn{2}{c|}{ QT-L } & \multicolumn{2}{c|}{ SPIHT } & \multicolumn{2}{|c|}{ JPEG2000 } & \multicolumn{2}{c|}{ HEVC } \\
\cline { 2 - 13 } & Coding & Decoding & Coding & Decoding & Coding & Decoding & Coding & Decoding & Coding & Decoding \\
\hline Girl & 0.0018 & $0.0395 \times 10^{-3}$ & 0.1245 & 0.0907 & 0.0390 & 0.0225 & 0.0155 & 0.015 & 40.6500 & 0.3970 \\
\hline 3DVenus & 0.0019 & $0.1390 \times 10^{-3}$ & 0.7578 & 0.7278 & 0.2783 & 0.2016 & 0.1205 & 0.0385 & 404.2500 & 3.5430 \\
\hline Horse & 0.0017 & $0.1287 \times 10^{-3}$ & 0.2867 & 0.2756 & 0.33 & 0.0892 & 0.0672 & 0.0542 & 125.664 & 1.2075 \\
\hline Average & $\mathbf{0 . 0 0 1 8}$ & $\mathbf{0 . 1 0 2 4} \times 10^{-3}$ & $\mathbf{0 . 3 8 9 7}$ & $\mathbf{0 . 3 6 4 7}$ & $\mathbf{0 . 2 1 5 8}$ & $\mathbf{0 . 1 0 4 4}$ & $\mathbf{0 . 0 6 7 7}$ & $\mathbf{0 . 0 3 5 9}$ & $\mathbf{1 9 0 . 1 8 8}$ & $\mathbf{1 . 7 1 5 8}$ \\
\hline
\end{tabular}

the wavelet-based and HEVC codecs, the full processing time is highly influenced by the resolution of the test hologram. When comparing the coding delays of the four codecs, we can plainly discern that HEVC is very time-consuming compared to the other three tested codecs. This is not surprising since the quad-tree structured coding units and the Inter prediction with high number of modes, that characterise HEVC coding technique, cause high computational complexity. Note that in our simulation we used the HEVC Test Model 16.9 of HEVC. On the other hand, JPEG-OPT codec produces the fastest encoding and decoding times. On average, the JPEGOPT encoder is 35 times faster than JPEG 2000, 83 times faster than SPIHT and 232 times faster than QT-L, which is more than fast enough for real-time applications.

\section{Overview of the analysis of simulation experiments}

To summarize, the results of the conducted experiments we can plainly discern that:

- In most cases, HEVC produces the best quality. But this is at the expense of extremely high coding and decoding time.

- JPEG 2000 requires the lowest encoding and decoding times among the wavelet-based codecs.

- QT-L is the slowest among the wavelet-based codecs and often produces Delta-PSNR results comparable to those obtained with SPIHT.

- JPEG-OPT yields very competitive compression performance and reconstruction quality that are sometimes even better than those of wavelet-based techniques.

As a final note, it is obvious that fast encoding/decoding is a crucial task in modern low-complexity applications such as embedded systems. In addition, JPEG-OPT coding scheme inherently supports backward and forward compatibility with legacy JPEG formats, which is not the case for wavelet-based codecs.

\section{CONCLUSIONS}

In this paper, we explore the efficiency of a DCT-based coding scheme for the compression of digital holograms. The proposed codec uses a Lagrangian-driven rate allocation mechanism while still compliant with the JPEG bitstream. Regarding compression efficiency and reconstruction quality, it yields competitive results with wavelet-based codecs that also include rate/distortion optimisation mechanism. Moreover, an empirical complexity evaluation showed that it is 37 times faster that JPEG 2000. Finally, we believe that contrarily to DH specific coding solutions in the literature, the optimized JPEG coding scheme offers a good balance of speed, file size and reconstruction quality. Since it guarantees backward compatibility with legacy JPEG decoders, it may serve as an attractive alternative to the current state-of-the-art and that the conclusions derived from the conducted studies are helpful for developing new JPEG-based hologram codec in line with the objectives of the upcoming JPEG Pleno standard.

The research done in this work could be extended by using perceptual metrics. Since the final destination of a compressed hologram is often to be displayed to a human observer, the perceived visual quality should be the criterion to take into account when evaluating a compression algorithm.

\section{ACKNOWLEDGMENT}

The authors are particularly grateful to David Blinder and Peter Schelkensa, (ETRO-VUB, Brussels, Belgium), Manuella Pereira (University of Beira Interior, Covilha, Portugal), José Peixeiro (Técnico Lisboa, Portugal) and Patrick Gioia (Orange Labs, Rennes, France) for their support, the very interesting discussions and for giving us access to their holographic display.

\section{REFERENCES}

[1] D. Gabor, "A new microscopic principle," Journal of Nature, vol. 161, 1948.

[2] F. Yaras, H. Kang, and L. Onural, "State of the art in holographic displays: A survey," IEEE Journal of Display Technology, vol. 6, no. 10, pp. 443-454, Oct. 2010.

[3] G. p. Tricoles, "Computer generated holograms: an historical review," Applied Optics, vol. 26, no. 20, pp. 4351-4360, Jan. 1987.

[4] A. Symeonidou, D. Blinder, A. Munteanu, and P. Schelkens, "Computergenerated holograms by multiple wavefront recording plane method with occlusion culling," Optics Express, vol. 23, no. 17, 2015.

[5] T. J. Naughton, Y. Frauel, B. Javidi, and E. Tajahuerce, "Compression of digital holograms for three-dimensional object reconstruction and recognition," Optics Express, vol. 41, no. 20, pp. 4124-4132, Nov. 2002.

[6] R.L. Graham, "An Efficient Algorithm for Determining the Convex Hull of a Finite Planar Set," Information Processing Letters, vol. 1, pp. 132133, 1972.

[7] E. Darakis, T. Naughton, J. Soragha, and B. Javidi, "Measurement of compression defects in phase-shifting digital holographic data," in Proceedings of SPIE, Optical Information Systems IV, vol. 6311, San Diego, California, United States, 2013.

[8] Y. Xing, B. Pesquet-Popescu, and F. Dufaux, "Comparative study of scalar and vector quantization on different phase-shifting digital holographic data representations," in 3DTV-Conference: The True Vision - Capture, Transmission and Display of $3 D$ Video. Budapest, Hungry: IEEE, mar 2014. 
[9] Y. Xing, M. Kaaniche, B. Pesquet-Popescu, and F. Dufaux, "Digital holographic data representation and compression," Academic Press, Oct. 2015.

[10] F. Dufaux, Y. Xing, B. Pesquet-Popescu, and P. Schelkens, "Compression of digital holographic data: an overview," in SPIE Proc. Applications of Digital Image Processing XXXVIII, San Diego, CA, USA, Aug. 2015.

[11] A. Arrifano, M. Antonini, M. Pereira, "Multiple description coding of digital holograms using Maximum-a-Posteriori," in IEEE 4th European Workshop on Visual Information Processing (EUVIP), Paris, France, 2013.

[12] D. Blinder, A. Ahar, A. Symeonidou, Y. Xing, T. Bruylants, C. Schreites, B. Pesquet-Popescu, F. Dufaux, A. Munteanu, and P. Schelkens, "Open access database for experimental validations of holographic compression engines," in Proc. 7th International Workshop on Quality of Multimedia Experience (QoMEX'2015), Messinia, Greece, May 2015.

[13] J. Peixeiro, C. Brites, J. Ascenso, and F. Pereira, "Digital holography: Benchmarking coding standards and representation formats," in IEEE International Conference on Multimedia and Expo (ICME). Seattle, WA, USA: IEEE, 2016.

[14] JPEG PLENO Abstract and Executive Summary, ISO/IEC JTC 1/SC 29/WG1 N6922, Sydney, Australia, 2015.

[15] V. Ratnakar and M. Livny, "RD-OPT : An efficient algorithm for optimizing DCT quantization tables," in IEEE Processings of the Data Compression Conference (DCC). Snowbird, UT, USA, USA: IEEE, mar 1995, pp. 332-341.

[16] G. K. Wallace, "The JPEG still picture compression standard," Соттиnications of the ACM, vol. 34, no. 4, pp. 30-44, Apr. 1991.

[17] A. Munteanu, J. Cornelis, G. V. der Auwera, and P. Cristea, "Wavelet image compression - the quadtree coding approachs," IEEE Transactions on Information Technology in Biomedicine, vol. 3, no. 3, pp. 176-185, 1999.

[18] A. Skodras, C. Christopoulos, and T. Ebrahimi, "The JPEG 2000 still image compression standard," IEEE Signal Processing Magazine, vol. 18, no. 5, pp. 38-58, Sep. 2001.

[19] A. Said and W. Pearlman, "A new fast and efficient image codec based on set partitioning in hierarchical trees," IEEE Transactions on Circuits and Systems for Video Technology, vol. 6, no. 3, pp. 243-250, Jun. 1996.

[20] G. J. Sullivan, J.-R. Ohm, W.-J. Han, and T. Wiegand, "Overview of the High Efficiency Video Coding (HEVC) Standard," IEEE Transactions on Circuits and Systems for Video Technology, vol. 22, no. 12, pp. 16491668, Dec. 2012.

[21] G. BjØntegaard, "Calculation of average PSNR differences between RDcurves," Technical Report VCEG-M33, ITU-T SG16/Q6, Austin, Texas, USA, 2001. 